\title{
Allometric Laws in Modular Dynamics: The Bauplan of Ontogenesis
}

\author{
Peter L. Antonelli ${ }^{1}$, Solange F. Rutz ${ }^{2, *}$ \\ ${ }^{1}$ Department of Mathematical and Statistical Sciences, University of Alberta, Edmonton, Canada \\ ${ }^{2}$ Department of Mathematics, Federal University of Pernambuco, Recife, Brazil
}

\section{ARTICLE INFO}

Article History

Received 19 September 2019

Accepted 04 November 2019

\begin{abstract}
In recent decades, a resurgence of allometry in ecology and its associated scaling laws has been observed, going under the name macroecology. It is reasonable to think that the plethora of current works on experimental physiology using allometry is a continuation of the tradition of searching for the "Holy Grail" or Bauplan, the foundation of organic form and metabolic function. The project our group focused on over several decades is the development of a corpus of geometric techniques, especially Finsler differential geometry, for the study of systems of second order ordinary differential equations called Analytical Modular Dynamics, which seeks to describe interactions between cell populations of various organs. Thus, the Huxley/Needham Law in allometry becomes a consequence of metabolism and physiological interactions. The models obtained contrast strongly with Riemannian theory. The geodesic coefficients for our example depend only on the $x$-variables, as in all Riemannian geometries, but, is true in Finsler theory only for Berwald spaces.
\end{abstract}

(C) 2020 The Authors. Published by Atlantis Press SARL.

This is an open access article distributed under the CC BY-NC 4.0 license (http://creativecommons.org/licenses/by-nc/4.0/).

\section{INTRODUCTION: THE RISE OF ALLOMETRY}

Recently, there has been a flurry of excitement in ecology concerning the resurgence of allometry and its associated scaling laws going under the name macroecology [1]. The 20th century works on allometry by Huxley [2], Needham [3], Laird [4], and Medawar [5], have formed a basis for ontogenic studies of large numbers of fauna, which ultimately has informed this recent interest. It is reasonable to think that the plethora of current works on experimental physiology using allometry is a continuation of the tradition of searching for the "Holy Grail" or Bauplan, the foundation of organic form and metabolic function.

Sir Joseph Needham called his discovery of allometry in his experimental studies of growing animal embryos, a "chemical ground plan" for development [3]. Also, Sir Julian Huxley, father of the Neo-Darwinian paradigm, focused on allometric descriptions of morphology in animals, especially invertebrates [2], and Laird [4] proved the ubiquity of Gompertz growth curves for organ biomasses in large numbers of vertebrate species. She discovered that virtually all organ biomasses of a given individual have the same Gompertz rate constant. Although these are different for different individuals, they are characteristic of specific species. She concluded that the Huxley/Needham straight line allometric law holds for many sizes and kinds of vertebrate individuals.

Also in plants allometry is a major topic. Allometry in Plants: The Scaling of Form and Process [6], presents an important engineering

“Corresponding author. Email: solange.rutz@gmail.com perspective on a "design of plants" based on allometry between their multitudes of organs. Such studies can be seen to relate historically to the 19th century work on the morphology of plants by von Goethe [7] with his botanist contemporary, de Candolle and Agnes's work in the 1950's [8]. Application in plants was slow. Yet, allometry was used in American forestry as a method for estimating crown-biomass from trunk diameter in stands of trees, by the 1940's [9]. Before that, allometry with respect to scaling and as differential growth was discussed in On Growth and Form, by Thompson [10].

Although, Harper's 1967 magnum opus [11], on plant ecology brought flora into mathematical and quantitative ecology in a way comparable to what had already been achieved for fauna, no special role had been singled out for allometric ideas. However, Harper in 1976 wrote "it appears therefore that the 3/2 thinning law describes an upper limiting condition which may not be exceeded by any combination of surviving plant numbers and weights" [12]. In Harper's concept of plants as clonal organisms, the modular unit or phytomer, is a piece of stem, a bud and a subtending leaf and this is repeated throughout the development and lifetime of plants. The individual phytomers may, in the vegetative phase, consists of vegetative leaves, whereas in the generative phase petals and stamen can be considered as variations on leaves. This great finding of von Goethe has been corroborated by molecular biology.

In the past two decades, allometry has been an active field of research in particular because of the West-Brown-Enquist model $[1,13]$. Moreover, datasets have become increasingly large, also in plants. In a study on scaling relationships between leaf area and leaf shape in 12 species of Rosaceae, more than 3000 leaves were sampled, and for each leaf 500 data points were determined [14]. 


\section{ALLOMETRY AND HILBERT'S FOURTH PROBLEM}

If we think of allometry as simply a collection of straight lines obtained by least squares fitting of pairs of data points on morphology, physiology, or ecology for individuals, or populations, with a single regression line for each data pair, then allometry becomes a sort of species-specific blue-print of internal architecture, or of external architecture as observed in plant phyllotaxis [15], the forms of flowers or in seashells [16]. The method has been applied for decades to non-biological objects. There is for instance, the famous "golden ratio" approach to fine art and architecture [17]. There are also the lesser known Gutenberg-Richter Law for the frequency distribution of earthquakes, the Pareto Law for the distribution of incomes among individuals, the so-called Zipf's Law for the sizes of cities and the Kleiber Law for the basal metabolic rate in individual animals, especially humans [18].

The project our group focused on over several decades is the development of a corpus of geometric techniques, especially Finsler differential geometry, for the study of systems of second order ordinary differential equations (SODE's) called Analytical Modular Dynamics (AMD), which seeks to describe interactions between cell populations of various organs, each producing hormones, $x$ 's, effecting the set of organs in an individual during growth and development, and which exhibit the Huxley/Needham allometric law between the $x$ 's produced. Thus, the Huxley/Needham Law becomes a consequence of metabolism and physiological interactions describing the dynamics of hormone production in the different sets of modular units (i.e., cells of organs). We have also developed a theory of Brownian motion in Finsler geometry in order to model internal noise during development [19].

Readers who are not familiar with Finsler geometry may recall the helpful dictum, "Finsler geometry is Riemannian geometry without the quadratic restriction", uttered by the great geometer, Chern, at the first AMS conference on Finsler Geometry, in 1995, in Seattle, Washington. It refers to the scalar product on tangent spaces of Riemannian manifolds being allowed relaxation from this quadratic condition to become a norm on tangent spaces of Finsler manifolds.

One important finding in Finsler science is the equivalency to Hilbert's 4th problem, that of classifying the Finsler geometries having straight lines as shortest distances between two points, where straight lines are allometries holding globally [20-22]. The mathematician, Berwald, founder of Finsler geometry (along with Cartan and Finsler), is credited with solving this famous problem in twodimension, with the condition that the geodesic equations be quadratic, called nowadays, geodesics of Berwald space [23]. The general case remains unsolved to this day.

\section{ANALYTIC MODULAR DYNAMICS}

One proceeds in AMD by modelling a dictum from 19th century Russian botanists studying lichen symbiosis [24]. In the deep evolutionary past of lichens, the algal and fungal partners interacted ecologically and gradually that interaction became more and more integrated due to genome modifications, thereby stabilizing chemical exchanges [24]. Following the early work of Volterra, Gause, Witt and Lotka, it is natural to try constant coefficient quadratic equations to model this. But this must be coupled with chemical production, so that over time, these coefficients become dependent on the products the alga and fungi produce. Furthermore, the symbiosis must have energy constraints and so SODE candidates must be Euler-Lagrange equations for a cost function, $F$, depending on $x, d x$, and $t$.

Furthermore, the cost must be assumed to be first degree positively homogeneous in $d x$ (just the norm condition mentioned above) so that the total cost over a time interval will be independent of how time is measured. Moreover, if one assumes each partner reproduces its modular units at nearly the same rate (so each kind of cell is never isolated from the other), reparametrization of production curves with $S$, with $d S=F(x, d x)$, eliminates, $t$. In this way the EulerLagrange equations become geodesics of the Finsler geometry defined by $F$. If the assumption of quadratic $F$-geodesics (i.e., geodesics of Berwald spaces) is adhered to the two-dimensional Finsler geometries possible are essentially of three types and when the geodesic coefficients are all constants, are described by the theorem known as the Finsler Gate [19-21; Appendix]. Such constant connection Berwald spaces have ecological meaning and broad applicability in ecology, evolution, physiology and epidemiology [20-23,25].

Among the three Berwald types one has an allometric Bauplan, i.e., has geodesics that are straight lines as in Hilbert's fourth problem mentioned above. To briefly describe Berwald's idea, we write

$$
F\left(x, \frac{d x}{d t}\right)=\frac{1}{2}\left[N^{1} H_{1}+N^{2} H_{2}\right] \text { where } H_{1}=\frac{F}{N^{1}}, \text { and } H_{2}=\frac{F}{N^{2}}
$$

Then, taking first order Taylor expansions by $N^{1} / N^{2}$ of $H_{1}$ and by $\mathrm{N}^{2} / \mathrm{N}^{1}$ of $\mathrm{H}_{2}$, we arrive at the symmetric first order approximation of the cost $F$ :

$$
\frac{d S}{d t}=\frac{N^{1}}{2}\left[P_{0}(x)+P_{1}(x) \frac{N^{1}}{N^{2}}\right]+\frac{N^{2}}{2}\left[Q_{0}(x)+Q_{1}(x) \frac{N^{2}}{N^{1}}\right]
$$

If we stipulate that $P_{0}=4 Z(x), P_{1}=2, Q_{0}=2 Z^{2}(x)$ and $Q_{1}=0$, the $F$-geodesics conform to the Huxley/Needham allometric law, provided $x=\left(x^{1}, x^{2}\right)$ are interpreted as log biomasses and $Z(x)$ is a smooth solution of Berwald's equation for projective flatness, $Z \partial_{1}(Z)=\partial_{2} Z[25]$. Here the subscripts indicate partial differentiation by either $x^{1}$ or $x^{2}$. Rewriting, we have the Kropina-type Finsler metric expression

$$
\frac{d S}{d t}=\frac{\left(N^{1}+Z(x) N^{2}\right)^{2}}{N^{2}}
$$

There are many $Z(x)$ that provide solutions and have positive Berwald-Gauss curvature scalar indicating Jacobi stability of solution trajectories $[26,27]$. A simple Riemannian geometric example of this type of stability is great circle arcs on a sphere. They oscillate back and forth crossing any chosen arc at the poles. Geodesics on a trumpet-shaped surface diverge away and so the system, having negative curvature is Jacobi unstable. Finally, along any solution curve of the Kropina metric the Huxley/Needham law holds true provided Berwald's projective equation holds. 


\section{CONCLUSION}

We have used the above procedure to obtain interaction schemes whose Finslerian cost functionals depend explicitly on the ratios $N^{1} / N^{2}$ while maintaining the Huxley/Needham law along solutions. The Euler-Lagrange curves are geodesics of the Kropina metric above [27]. The curvature scalar is highly variable. This contrasts strongly with Riemannian theory where the projective geometries must have constant curvature. The geodesic coefficients for our example depend only on the $x$-variables. Of course, this holds for all Riemannian geometries, but, is true in Finsler theory only for Berwald spaces [23].

\section{CONFLICTS OF INTEREST}

The authors declare they have no conflicts of interest.

\section{REFERENCES}

[1] Brown JH, Gillooly JF, Allen AP, Savage VM, West GB. Toward a metabolic theory of ecology. Ecology 2004;85;1771-89.

[2] Huxley JS. Problems of relative growth. 2nd ed. New York: Dover Press; 1972.

[3] Needham JA. Heterogony, a chemical ground-plan for development. Biol Rev 1934;9;79-109.

[4] Laird AK. Dynamics of relative growth. Growth 1965;29;249-63.

[5] Medawar PB. The growth, growth energy, and ageing of the chicken's heart. Proc R Soc Lond B 1940;129;332-55.

[6] Niklas KJ. Plant allometry: the scaling of form and process. Chicago: University of Chicago Press; 1994.

[7] von Goethe JW. Goethe's botany: the metamorphosis of plants. Sacred Science Library; 1790.

[8] de Candolle A, Agnes A. The natural philosophy of plant form. Cambridge University Press; 1950.

[9] Kittredge J. Estimation of the amount of foliage of trees and stands. J Forestry 1944;42;905-12.

[10] Thompson DW. On growth and form: the complete revised Edition. Dover Press; 1942.

[11] Harper JL. Population biology of plants. London: Academic Press; 1977, p. 892.
[12] May R, McLean AR. Theoretical ecology: principles and applications. 3rd ed. Oxford University Press; 2007.

[13] West GB. Scale: the universal laws of growth, innovation, sustainability, and the pace of life in organisms, cities, economies, and companies. Penguin Press; 2017.

[14] Yu X, Hui C, Sandhu HS, Lin Z, Shi P. Scaling relationships between leaf shape and area of 12 Rosaceae species. Symmetry 2019;11;1255.

[15] Jean RV. Phyllotaxis: a systemic study in plant morphogenesis. New York: Cambridge University Press; 1994, p. 386.

[16] Meinhardt H. The algorithmic beauty of sea shells. Springer Science \& Business Media; 2009.

[17] Dunlap RA. The golden ratio and Fibonacci numbers. World Scientific Press; 1997.

[18] Kleiber M. Body size and metabolic rate. Physiol Rev $1947 ; 27 ; 511-41$

[19] Antonelli PL, Zastawniak TJ. Fundamentals of Finslerian diffusion with applications. Dordrecht: Springer; 1999.

[20] Antonelli PL, Leandro CG, Rutz SF. Phenotypic plasticity for allometric laws of ontogeny from cellular interactions. Univ J Appl Math Comput 2015;3;37-53.

[21] Antonelli PL, Leandro CG, Rutz SF. Phenotypic deformation: the role of allometry and the golden ratio. Int J Appl Math 2016;29;485-503.

[22] Antonelli PL, Leandro CG, Rutz SF. Stochastic canalization of phenotypic deformations during ontogenesis. Int J Appl Math 2016;29;655-71.

[23] Antonelli PL. Berwald space, Encyclopedia of Math. Available from: http://www.encyclopediaofmath.org/index.php?title= Berwald_space \&oldid=12731.

[24] Khakhina LN. Concepts of symbiogenesis: a historical and critical study of the research of Russian botanists. Yale University Press; 1992.

[25] Antonelli PL, Rutz SF, Hirakawa CE. The mathematical theory of endosymbiosis I. Nonlinear Anal Real World Appl 2011;12;3238-51.

[26] Antonelli PL, Bradbury RH. Volterra-Hamilton models in the ecology and evolution of colonial organisms. Singapore: World Scientific Press; 1996, p. 228.

[27] Antonelli PL, Ingarden RS, Matsumoto M. The theory of sprays and Finsler spaces with applications in physics and biology. Netherlands: Springer; 1993. 